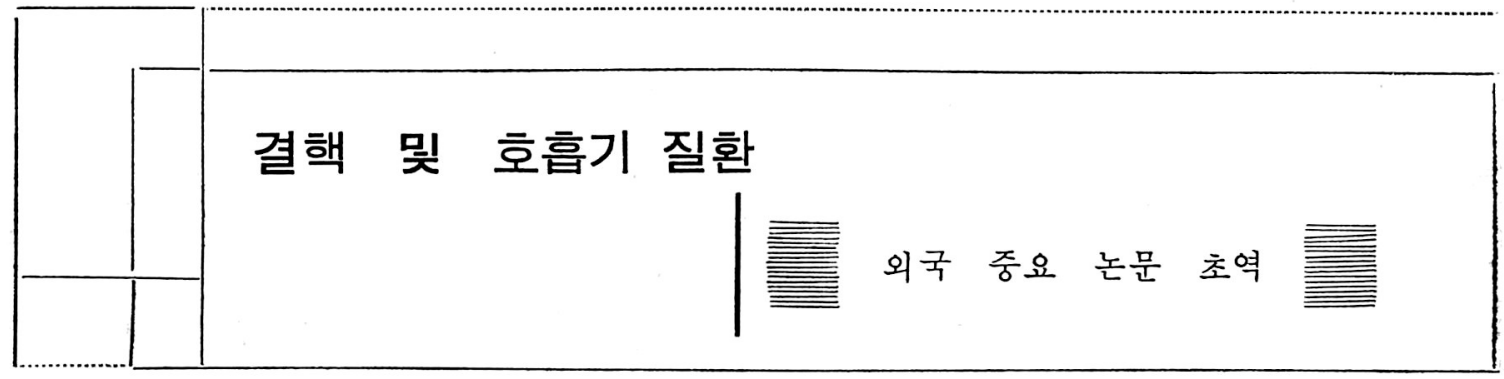

結 核疫學 의

\section{Dynamic Model}

\section{A Dynamic Model for the Epidemiology of Tuberculosis}

\section{by Hans T. Waaler}

Amer. Rev. Resp. Dis.,

Vol.98, No. 4 Oct. 1968

抗結核問題를 가장 效果的으로 遂行하는데 있어서 여 러가지 부합되는 측정방법은 주어진 豫算으로서 最小限 의 問題點으로 축소시켜야 理想的이나.

사실 結核에 對한 完全化된 Dynamics 란 여러가지 측 정도의 양상, 장기간의 항결핵 작용의 반향, 낙관적인 문제에 대한 時代的인 評價에 따라서 그 양상을 다소 달 리하므로 결코 쉬운 문제가 될 수는 없다.

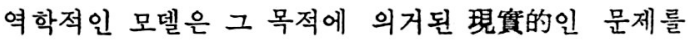
해결하기 위해 상세하게 실질적으로 되어야 하며 기술 적인 문제에 있어 Computer 를 사용하여 신속하게 처리 해야 할 것이다.

여기 소개되는 모델은 초반기 度學的인 문제와 Dynamic 한 點을 연관지우며, 간격을 두고 보는 將來의 疫 學的인 문제를 예시함은 勿論 그 傾向을 推算하게 한 다.

\section{모델의 基本的인 構造}

(Basic Structure of the Model)

모델의 重要한 要素의 하나는 어떤 限定되는 人口인 데 이것은 疫學的인 有意性에 따라서 몇개의 상호 유기 적인 계급군(class)으로 區分될 수 있다. 계급군의 구성
은 결핵의 natural dynamics 때문에 계속적으로 變化할 수 있다. 즉 결핵의 natural dynamics 란 減染, 死亡, 羅患, 治癒再發, specific antituberculosis measure 로서 BCG, 患者發見, 治療, 그리고 demographic factor 로서 出生, 死亡 年龄이 重要한 要素가 된다. migration 은 이 모델에서 除外되었다. 現在의 모델은 人口群과 그젓의 subgroup 이 不連續繁에 있음을 보이고 있다. 여기서 Dynamics 란 여러가지의 epidemiologic class 사이의 사 람에 對한 變化率(確率)을 意味하게 된다. 솟 class 는 Age-specific 한 것이머 epidemiologic transfer 가 年䍅에 따라서 同時에 代置할 수 있다.

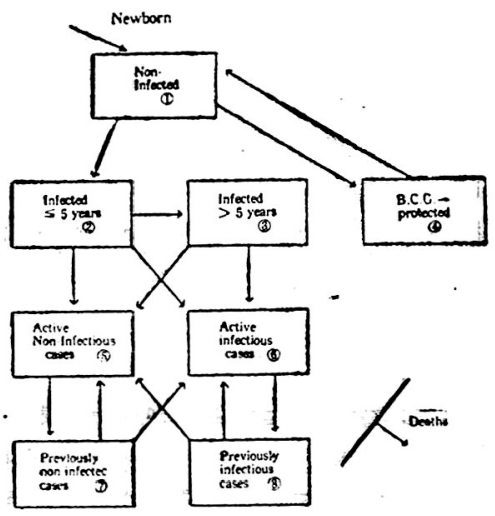

모델은 서로 다른 方程式組를 이루고 있다. 또한 방 정식이 二次방정식으로 되어 있기 때문에 明確한 分析 結論은 얻기가 난이하다.

\section{疫舅級群에 對한 解說}

(Description of the Epidemiologic Classes)

疫學的 級群은 Definition 에 따라서 여러가지로 區分 되어 진다. 여기에 적용된 Definition 은 統計學과 놀웨 이의 統計學的 傳統에 많은 영향을 입었다. 즉 예를 들 면 活動性患者의 中間的 分類로서 傳染性인 것 과 非傳染 性인것으로 나눌 수 있는데 이것은 塗抹檢查에 依한 菌 
결핵 및 호흡기질환 Vol.16, No.1, January 1969

陽性과 培盖에서만 菌陽性으로 나타남으로서 區分되는 것이다.

疫學的 級群은 대충 8區分 되는데 즉 1. Non infected, 2. Infected( $\leq 5$ years), 3. Infected(>5years) 4. BCG Protection 5. Active Non-infectious cases 6. Active Infectious cases 7. Inactive Previously Non-infectious cases,

8. Inactive Previously Infectious cases.

1. The non-infected: 減染이 自由로워질 수 있는 사 람들인데 結核菌에 依해서 그 양상이 달라진다. 이것은 투버큐린반응 검샤로서 減染者를 推算할 수 있다. 그러 나 高令層의 陰性, 투버큐린檢查의 오류, 낮은 反應度 에 制缽의 문제가 수반된다.

2. The infected: 減染은 時間에 따라서 상당한 영향 을 받게 되는데 이것은 減染以後 5 年以上의 경우와 5 年 以下의 경우로 나누어 지는데 그 重要한 理由로는 罹患 率이 時間의 경과에 依해 상당한 영향을 끼치기 때문이 다.

3. Person protected by B.C.G. : 完全히 면역을 획득했 다고 볼 수 있는 B.C.G 接種을 받은 사람을 말한다. B.C. $G$, 接種에 있어서 $3 \%$ 의 接種率로서 $\mathrm{P} \%$ 의 Protection 이 있다면 疫學的 假定으로 同一한 疫學的인 效果値 $\mathrm{C} \times \mathrm{P}$ 는 一定하게 유지하게 된다.

4. Active cases : active cases 는 活動性 非傳染性 경 우와 活動性 傳染性인 경우로 區分된다. 傳染性與否에 따라서 이렇게 나누어 지는데 여기에서 治療의 化學 療法도 그 方法을 달리하게 된다.

5. Inactive cases : 여기는 두가지 形態의 區分이 있게 되는데 하나는 Previously noninfectious subject 로 된 것 과 또 하나는 proviously infections 된 形態이다. 이렇게 區分하는 主原因은 再發率에 差異를 이루게 하고 또한 化學療法에도 다른 力法을 적용케 하는 것이다.

Infection : 感染者는 非感染群에서 感染群 (<five years) 으로 轉換된 사람을 말한다 이러한 感染群은 年令과地 域社會內의 感染力에 따라 양상이 나타나게 된다.

즉, Converters(age $i)=($ noninfected $[\operatorname{Age}(i-1)]-$ vaccinated $) \times$ Sustceptibility (age $[i-1) \times$ force of infection.

※ force of infection;

Total number of infectious patients Total population

Age-specific susceptibility 의 推算은 투버큐린反應 檢 查 포함한 representative survey 나 longitudinal survey, 또는 報告가 잘 되고 있는 地域社會 어떤 記錄을 가지고 計算해 낼 수 있다.

Morbidity : 感染群에서 患者群으로 轉換되어 患者狀態 에 있는 率을 말한다 活動珄 患者로 되는 경 우에 있어
Infected years<5years 에서 바로 傳染性 患者로 되는 形 態와 이것이 active non-infectious cases 로 되는 것과 Infected $>$ 5years 에서 傳染性 患者로 되는 경우가 있다. 羅患率은 地域社會의 感染力의 强度에 따라 많은 영 향을 끼치게 된다

Healing : 治癒는 自治然的인 것과 人工的인 것으로 나 누어 진다. 治療의 效果는 自然的治痖를 포함한 percentage로 나타난다

Relapse: 再發은 Inactive previously infectious case 가 Active case 로 되어진 경우를 말하는데 이것은 네가지 의 전환 경로로 나눌 수 있다. 첫째는 두가지 길은 非活 動性過去 傳染性 患者에서 Active infectious case 로 되는 경우와 Active non-infectious case 로 되는 경우가 있다. 둘째 두가지의 길은 非活動性過去非傳染性 患者에서 Active infectious case 로 되는 경우와 Acitve non-infectious case 로 되는 경우가 있다.

Death : Age specific death rate 와 survival rate 를 算 出하게 된다. Active case 群에는 logitudional survey

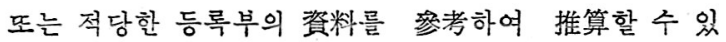
는 分離된 excess death rate 를 考慮하게 된다. 만약 excess death rate 를 $\mathrm{K}$ 로 표시하고 Age Specific survival 은 $\mathrm{Si}$ 라 하면 Survival rate: $1-\mathrm{K}(1-\mathrm{S} i)$ 로 표시 할 수 있다. 예로서 general survival rate 가 0.7 , excess death rate 가 2 라고 하면 이연령의 survial rate 는 1-2(1$0.7)=1-0.6=0.4$ 이다.

Calculation of New Pool Values.

1. 年齡别 過去傳染性患者 計算(Calculation of new values of "previous infectious cases age-group $i$ ") : 이 것은 再發하지 아니한 젊은 年齡層 $(1-i)$ 사람 즉, 그年 齢層 $(i-1)$ 의 活動性傳染患者中에서 治癒된 者로 構成 된다 이것을 슴하면 다음과 같이 group-specific survival rate 를 얻게된다.

$$
(1-\mathrm{Kt}[1-\mathrm{Si}-1])
$$

2. 年齡别 傳染性 患者計算(Calculation of new values of "active infectious cases in age-group $i$ ") : 이 群에 屬 하는 사람은 젊은 年齢屬 $(i-1)$ 에서 治癒되지 아니한 즉 어떤 年䍄群 $(i-1)$ 에서 過去傳染性에서 再發한 者, 過去非傳染性에서 再發한 者, 活動性非結核에서 더 惡 化된 者, 感染되어 新患으로 된 者, converter 에서 新患 으로 된 者를 構成으로 한다. 이러한 者들을 總合함으 로서 group-specific survival rate를 다음 公式으로 求할 수 있다.

$$
\left(1-\mathrm{K}_{2}[\mathrm{~S} i-1]\right)
$$

3. 結核問題争 計算(Calculation of the tuberculosis problem) : 이것을 측정하는 모델은 데이타의 substantial amount 를 주게 된다. Waaler 氏는 하나의 단순한 定義 
로서 苦痛과 經濟的 損失을 나타낼 수 있는 유용한 인 덱스로 取할 수 있도록 적용된다고 제시한 바 있다. 어 느 時期 $(\mathrm{t})$ 의 有病率을 Pt 라 하면 時間的인 문제에 연 관해서 有病率은 다음과 같이 計算한다.

$$
\mathrm{Pt}=f(\mathrm{t})
$$

문제는 또한 다음과 같이 ㅂ⽉ㅈㅈ되어 진다.

$$
\text { Problem }=\int_{0}^{\infty} \frac{\mathrm{Pt}}{(1+\mathrm{r}) \mathrm{t}} \mathrm{dt}
$$

여기서 $\mathrm{r}$ 는 장래 患者의 reduced importance 를 reflecting 하는 interest rate 를 말한다.

\section{疫學 모델에 따르는 要素}

1. 人口學的인 要素 : 몇 가지 데이타가 수반되어 진다 즉 (1) 5 歲 간격의 年齡別 人口數, (2)出生率(또는 出產 率) (3) 死亡率(年齡別로) (4) 活動性新患 및 過去患者에 적용되는 Excess mortality 等이다.

2. 疫學的인 要素: 대체로 다음과 같은 측정과 수치 가 있어야 한다. 즉 (1) 8가지 疫學群에 따라 Age-specific population. (2) 過去에 確示된 것으로서 “感染力”에 對 한 Susceptibility.

즉, Susceptibility $=\frac{\text { Sum of converters }}{\text { Force of infection }}$

(3) 두가지 Active case 에 있어서의 converter 에 한한 Morbidity. (4) 傳染性 患者에 對한 非傳染性 患者의 Progression rate (確率值). (5) 傳染性 吕 非傳染性患者의 治
療率. (6) 活動性 患者로 되는 再發率 等이다.

B.C,G program과 case finding, treatment program 도 重要한 문제로 다루어 진다. 더욱이 Incidence rate 는본 論文에는 그렇게 언급되지 아니 하였지만 結核瘦學에는 매우 重要한 것이다. 알아 두어야 할 Incidence rate 는 (1) Infection (2) new infectious 에 대한 Incidence (3) new non-infectious case 에 대한 Incidence. (4) Infectious case 의 再弡에 對한 Incidence (5) non-infectious case 의 再發 에 對한 Incidence 等이다.

$$
\text { (초역 자 房 基 交) }
$$

\section{References:}

1. Hans T. Waaler : A Dynamic Model for the Epidemiology of Tuberculosis, Amer. Rev. Resp. Dis. Vol. 98, No. 4 Oct., 1968.

2. Brogger, S. : Systems Analysis in Tubercuosis Control, A Model, Amer. Rev. Resp. Dis., 95 : 419, 1967.

3. Revelle, C.S., Lynn, W.R., and Feldman, F.: Mathematical models for the Economic $A$. lllocation of Tuberculosis Control Activities in Developing Nations, Amer. Rev. Resp. Dis., $96: 893,1967$. 